\title{
Designing Stainless Steel Reflector at VR-1 Training Reactor
}

\author{
Jan Frybort ${ }^{1, *}$, Pavel Suk ${ }^{1}$, and Filip Fejt ${ }^{1}$ \\ ${ }^{1}$ Department of Nuclear Reactors, Czech Technical University in Prague, V Holesovickach 2, 180 00, Prague, Czech Republic
}

\begin{abstract}
Light-water reactor cores are commonly surrounded by a stainless steel and water reflector. The reflectors are improving power distribution in the core, reducing the leakage of neutrons and thus also protecting the pressurized vessel from the neutron irradiation and the following embrittlement. Contrary to the standard procedures utilized for generation of the fuel assembly data, the reflector elements require a special approach. The major difficulty with the reflectors is represented by an absence of neutron sources in the reflector elements. Some artificial neutron source simulating the realistic source of neutrons from neutron leakage from the surrounding fuel assemblies must be added in the calculation model. The reflector data in the full-core calculations have a great impact on the power distribution in the core. The research in this field is usually focused on the square geometry, and therefore the accurate data for the hexagonal geometry are lacking. Improvements in this area are needed.

Training Reactor VR-1 is used for measurements related to nuclear engineering. Department of Nuclear Reactors operating this reactor at the Czech Technical University in Prague is currently designing reflector elements containing stainless steel in order to provide measurable characteristics that can be compared to calculations realized by either Monte-Carlo codes or macroscopic core simulators. This article summarizes the methodology of development of the reflector assemblies to improve their similarity with the VVER-1000 reflector. The impact of the evaluated nuclear data is assessed. Further improvements of the proposed design is necessary to reach better agreement with the neutron spectrum in VVER-1000 reactor reflectors. The influence of evaluated data on the global characteristics was found negligible.
\end{abstract}

\section{Motivation for Research}

The reflectors from water and stainless steel surrounding a reactor core reduce leakage of neutrons and decrease the reactor vessel damaging fast neutron flux. Currently, there is no definite methodology to generate the macroscopic data in the non-fuel regions available. Importance of the reflectors is two-fold. They influence both the reactor core reactivity and the power distribution in the core.

Training reactor VR-1 is used for measurements related to nuclear engineering and it is equipped with multiple experimental devices to study various phenomena related to the nuclear reactor operation. Department of Nuclear Reactors operating this reactor at the Czech Technical University in Prague is currently designing reflector elements containing stainless steel in order to provide measurable characteristics that can be compared to calculations realized by either Monte-Carlo codes or core simulators utilizing the macroscopic data.

\section{Training Reactor VR-1}

Training reactor VR-1 is a zero-power light-water reactor with an open pool. It facilitates core access and enables better awareness of the core components during education

*e-mail: jan.frybort@fjfi.cvut.cz and training. The fuel is in form of IRT-4M fuel assemblies. The fissile material mixture is $\mathrm{UO}_{2}$ mixed with Aluminum alloy in order to increase the temperature conductivity. It is not a limiting factor for the VR-1 reactor, but the same fuel is used in other experimental reactors with higher thermal power. Uranium is enriched to $19.7 \%$. The reactor core includes also other components for experimental purposes: vertical dry channels for irradiation and neutron flux measurement, graphite and beryllium reflector blocks, equipments for study of delayed neutrons, reactivity feedbacks, and activation analysis.

The great feature of the VR-1 reactor is its flexible core configuration. It is illustrated with two core layouts in figures 1 and 2 .

\section{Procedures}

The outcome of this study is the analysis and the improved design of the stainless steel containing reflector elements for the appropriate VR-1 core configuration. In order to compare the results for the VR-1 reactor with the VVER1000 reflectors [1], Monte-Carlo calculations of the 2D model of the VVER-1000 core from the available benchmark specification was conducted [2]. It was not only a static calculation but fuel depletion was conducted with average specific power for this type of reactors. This simplified burnup calculation was meant to asses the potential change of the power distribution in the core during the fuel 


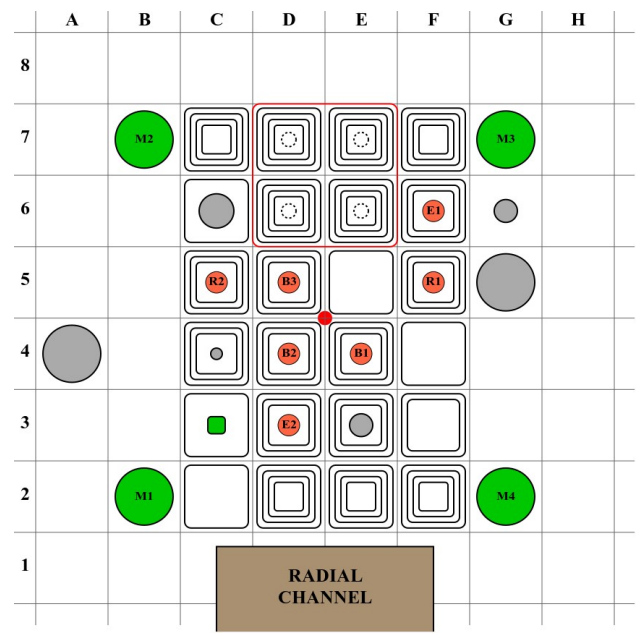

Figure 1: One example of potential VR-1 core configuration.

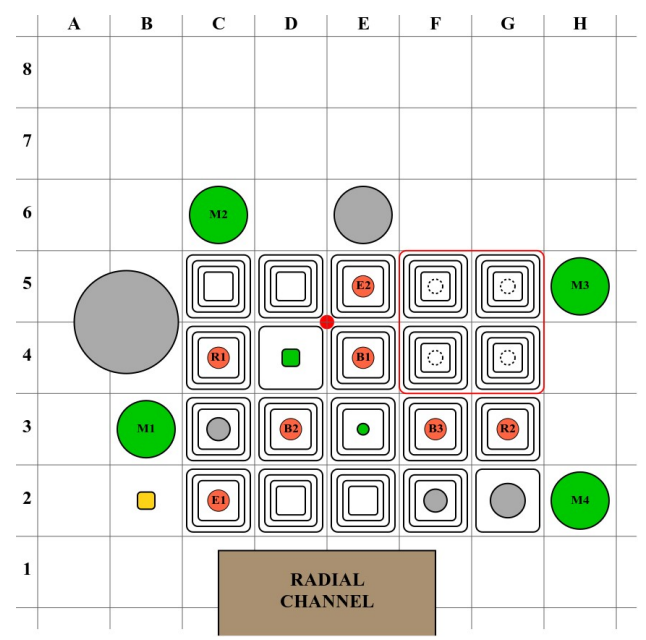

Figure 2: Second example of potential VR-1 core configuration.

irradiation. The fuel depletion was simulated by the Serpent Monte-Carlo code [3].

\section{VVER-1000 and VR-1 reflector configurations}

The VVER-1000 reactor is a pressurized-water reactor with 163 hexagonal fuel assemblies, each with 312 fuel pins, 18 guide tubes, and one central tube for instrumentation. The core is surrounded in the radial direction by a stainless steel reflector with water channels for cooling. There are several reflector types in the VVER-1000 core shown in figure 3 . All the calculations were conducted with ENDF/B-VII.1, ENDF/B-VIII.0 [4], and JEFF-3.3 data [5] in order to discover any dependence in this regard, but no significant differences were identified and result only for ENDF/B-VIII.0 will be presented.

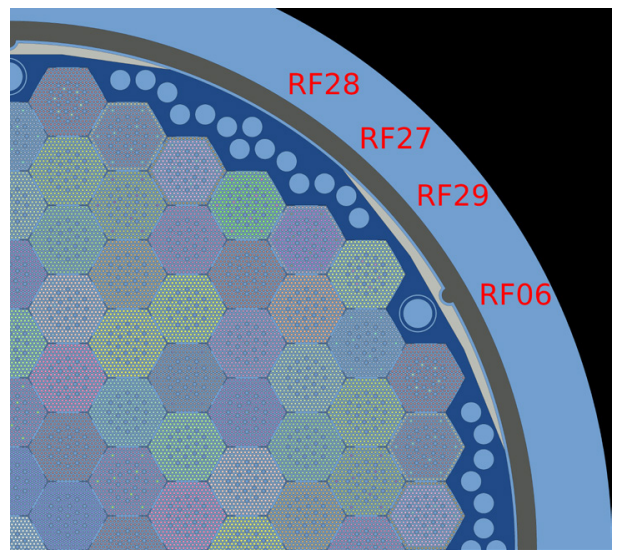

Figure 3: Reflector positions in VVER-1000 reactor.

Parameters of the potential VR-1 core reflectors were calculated by the Serpent Monte-Carlo code. A simplified core configuration was proposed for this analysis. It contains no vertical channels and no additional equipment. Plots from the calculations are shown in figure 4 for the reference blank core without the stainless steel reflectors and in figure 5 for the core with three reflector assemblies at one side of the core. The core configuration was chosen to resemble the VVER-1000 configuration.

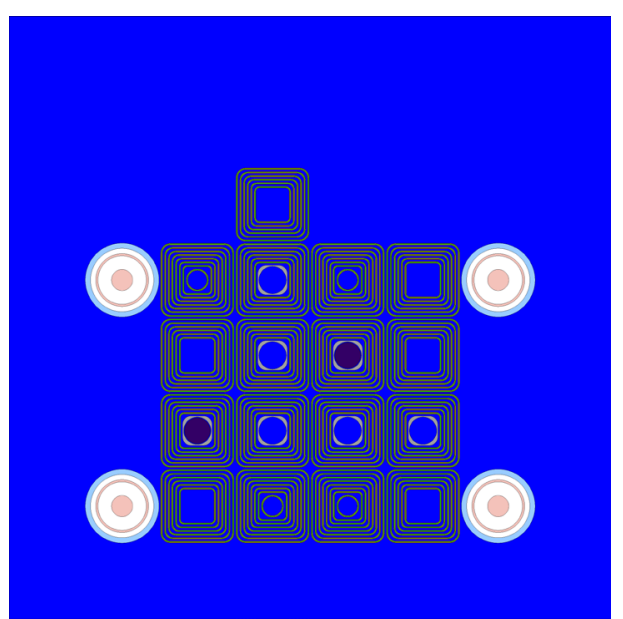

Figure 4: Core without stainless steel reflectors. 


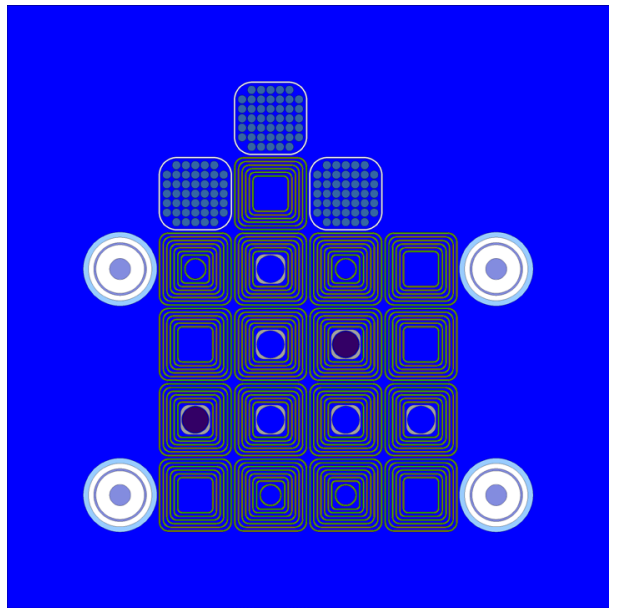

Figure 5: Core with stainless steel reflectors.

The VR-1 reflector assemblies are designed with the stainless steel rods with $8 \mathrm{~mm}$ in diameter in various latices and with or without a central dry channel for measurement. All the designs in this study are shown in figure 6 .

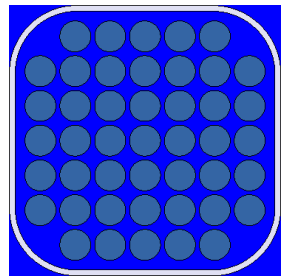

(a) Reflector design 1 .

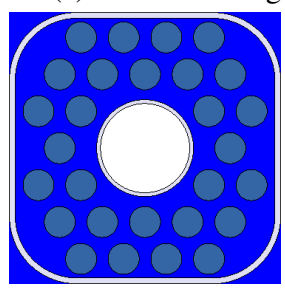

(c) Reflector design 3 .

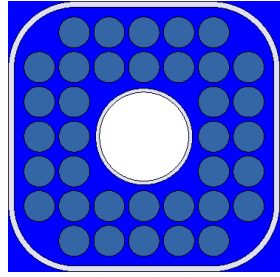

(b) Reflector design 2.

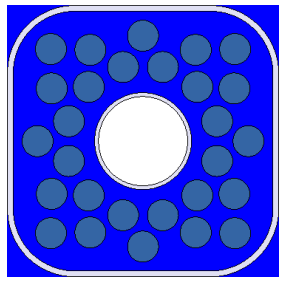

(d) Reflector design 4 .
Figure 6: Potential VR-1 reflector designs.

\section{Results}

The purpose of the study is to design reflectors that would produce a measurable response of the core characteristics. At this stage it is verified by calculations. The results are illustrated in the following figures.

\subsection{Flux distribution}

The global results are presented first in figures 7 and 8 . The less efficient neutron thermalization in the reflector assemblies is clearly visible from the thermal flux distribution shift.

The stainless steel reflectors are in the positions C6, D7, and E6 of the VR-1 reactor core. The numbering of positions is shown in figures 1 and 2 .

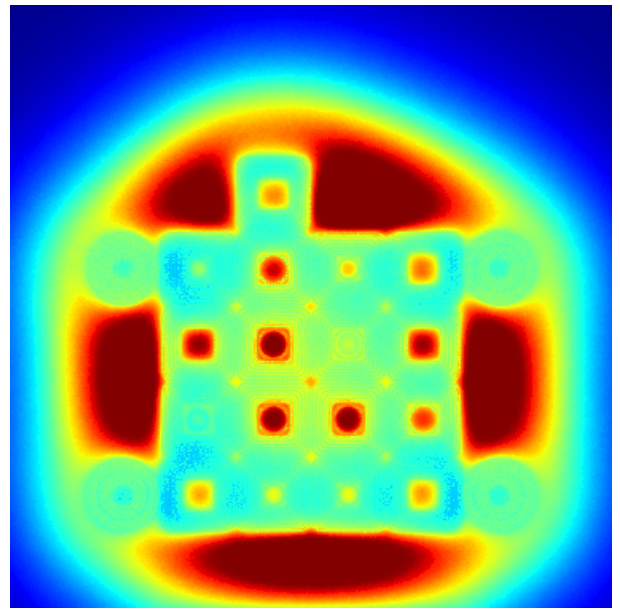

Figure 7: Thermal flux distribution in core without stainless steel reflectors.

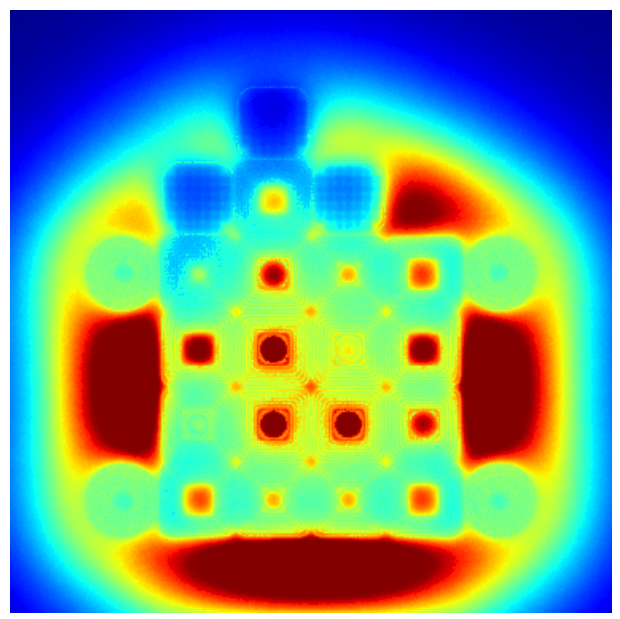

Figure 8: Thermal flux distribution in core with stainless steel reflectors.

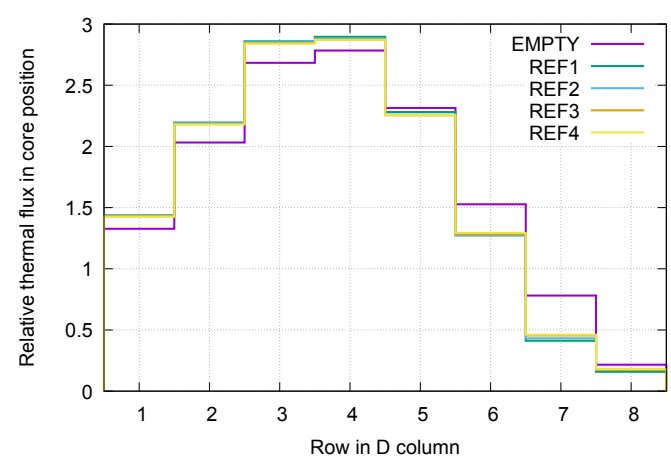

Figure 9: Detail of flux distribution shift.

Detail of the thermal flux distribution shift is shown in figure 9 for the column D of the VR-1 core. The less efficient neutron thermalization for the reflector assemblies results in the lower thermal neutron flux in the upper part of the reactor core. There are slight differences of the group fluxes among the reflector positions. It is plotted 


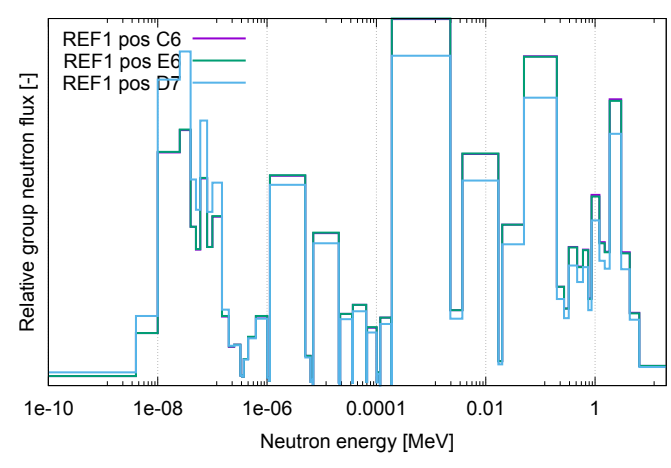

Figure 10: Groupwise neutron flux in reflector.

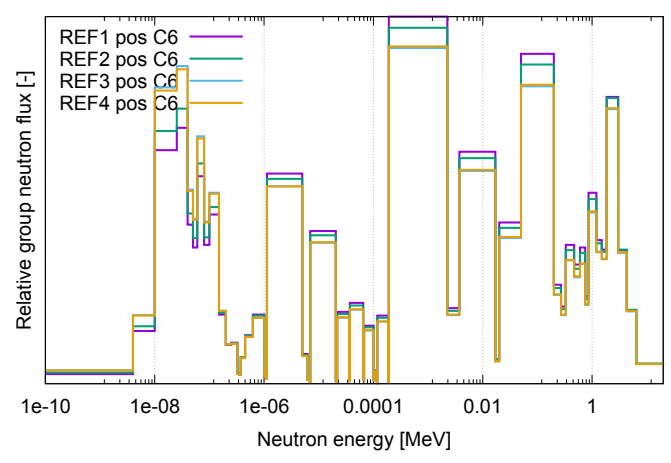

Figure 11: Neutron flux in position C6.

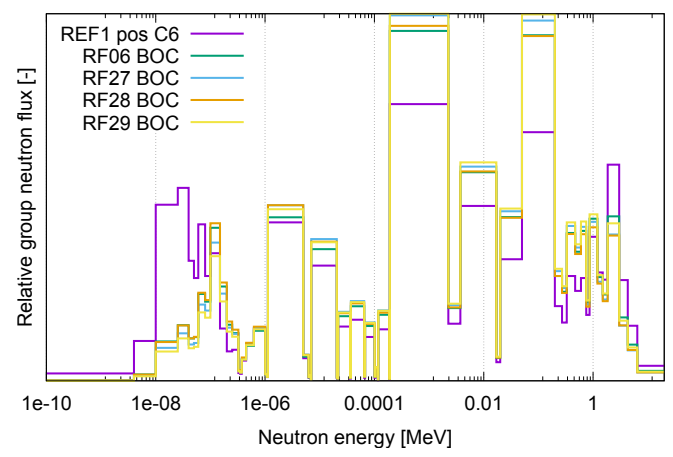

Figure 12: Difference between VVER-1000 and VR-1.

in figure 10. The performance of individual reflector designs from figure 6 is shown in figure 11. The high fast neutron flux is important for sufficient agreement between the reflectors in VR-1 and VVER-1000 reactors. The plot in figure 12 shows the differences between individual reflector types in the VVER-1000 core for the beginning of cycle (BOC) and the reflector design 1 in the position C6 in the VR-1 reactor.

\subsection{Future work}

It will be necessary to further improve agreement between the neutron fluxes in VVER-1000 and VR-1 reflectors.
The VR-1 core can be adjusted for this purpose. A more detailed analysis will conducted including partial neutron currents at reflector boundaries. Additionally, a sensitivity analysis will help to discover more detailed reflector characteristics.

\section{Conclusions}

Several designs of water and stainless steel reflectors were evaluated. It was proven that a substantial change in the VR-1 core characteristics can be achieved by their presence. It is result of the shift of thermal flux distribution in the core. The calculations revealed changes in the neutron spectra, the neutron flux distribution, the multiplication factor, and the reactivity worth of absorbing rods.

As a result of this project, the VR-1 reactor can contribute to the research of the techniques for generation of the macroscopic cross-section data for the non-fuel components of power nuclear reactors. It was found that the differences among the reflector designs are limited thus the next steps will be focused on improvement of the flux spectrum agreement with the VVER-1000 reflectors as it was determined by the Serpent calculations. After manufacturing and utilization in the VR-1 core the reflector assemblies at various configurations will enable the comparison between measurements, Monte-Carlo, and deterministic calculations.

This research was supported by the project no. CZ.02.1.01/0.0/0.0/16_013/0001790, financed from European structural and investment funds and Czech Republic funds.

\section{References}

[1] International Atomic Energy Agency, In-Core Fuel Management Code Package Validation for WWERs (IAEA, Vienna, 1995) 156 p.

[2] Krysl V., Mikolas P., Sprinzl D., Svarny J., "FullCore" VVER-1000 benchmark calculated by MOBYDICK macrocode (SKODA JS, Pilsen, 2015) 26 p.

[3] Leppänen J., Viitanen T., Valtavirta V. and Kaltiaisenaho T., The Serpent Monte Carlo code: Status, development and applications in 2013, Ann. Nucl. Energy 82, 142-150 (2015).

[4] Chadwick M. B., Herman M., Oblozinsky P., et al., ENDF/B-VIII.0: The 8th Major Release of the Nuclear Reaction Data Library with CIELO-project Cross Sections, New Standards and Thermal Scattering DataNuclear Data Sheets 148, 1-142 (2018).

[5] Santamarina A.,Bernard D.,Rugama Y., The JEFF3.1.1 Nuclear Data Library (OECD NEA, Paris, 2009) $62 \mathrm{p}$. 